\title{
PARENTALIDADE E DESENVOLVIMENTO SOCIOEMOCIONAL: UMA REVISÃO
}

\author{
PARENTALITY AND SOCIOEMOTIONAL DEVELOPMENT: A REVIEW
}

\author{
Jéssika dos Santos Alves ${ }^{1}$ \\ Islane Cristina Martins ${ }^{2}$
}

RESUMO: Sabe-se que as práticas parentais têm impacto no desenvolvimento infantojuvenil, entretanto, busca-se compreender qual o estilo parental apresenta práticas parentais mais favoráveis ao desenvolvimento das habilidades socioemocionais. Objetivo: Realizar uma revisão integrativa da literatura para encontrar evidências que possibilitem traçar o estilo parental que promove o desenvolvimento das competências socioemocionais de crianças e adolescentes. Material e Métodos: Foi feita uma busca das referências nas bases Periódicos CAPES, Google Scholar e Pubmed em abril de 2021. A busca permitiu identificação de 13 artigos que se adequaram aos critérios de elegibilidade. Resultados: Em 70\% dos artigos foi possível observar que o estilo parental indulgente como facilitador do desenvolvimento, nos outros $30 \%$ estilo autoritativo como vantajoso para desenvolvimento socioemocional. Conclusão: Portanto, foi possível observar que dois estilos parentais foram associados ao desenvolvimento socioemocional adequado: indulgente e autoritativo. Destacando que a afetividade tem maior impacto nas habilidades socioemocionais do que o rigor comportamental.

Palavras-chave: Competências Socioemocionais. Estilos Parentais. Inteligência Emocional.

ABSTRACT: It is known that parenting practices have an impact on child and young development, however, the search is to understand which parenting style presents parenting practices more favorable to the development of socio-emotional skills. Objective: To carry out an integrative literature review to find evidence that makes it possible to trace the parenting style that promotes the development of socialemotional skills in children and teenagers. Material and Methods: References were searched in the CAPES, Google Scholar and Pubmed journals in April 2021. The search allowed for the identification of 13 articles that met the eligibility criteria.

Results: In 70\% of the articles it was possible to observe that the indulgent parenting style as a facilitator of development, in the other $30 \%$ the authoritative style as advantageous for socio-emotional development. Conclusion: Therefore, it was

\footnotetext{
I Bacharel em Psicologia- Centro Universitário Jorge Amado -Salvador- Bahia, Brasil. ORCID: ooooooo2-7177-3313.E-mail:je.alvesi99o@gmail.com.

2 Doutora em Neurociências - Universidade Federal de Pernambuco, Brasil. ORCID: oooo-0003-23512730. E-mail: islanemartins@gmail.com.
} 
possible to observe that two parenting styles were associated with adequate socialemotional development: indulgent and authoritative. Emphasizing that affectivity has a greater impact on socio-emotional skills than behavioral rigor.

keywords: Emotional Intelligence. Parenting Styles. Socioemotional Skills

\section{INTRODUÇÃO}

É comum confundir a parentalidade e o estilo parental, a parentalidade é um conceito que retrata o movimento das figuras parentais para a construção de um ambiente seguro e uso de recursos ambientais e culturais disponíveis extra e intrafamiliar para promover o desenvolvimento infantil (PIRES COLTRO; PARAVENTI; VIEIRA, 2020).

Sendo assim, o estilo parental é um conjunto de comportamentos, atitudes e cognições do adulto direcionados para a criança, no qual cria um clima emocional. $O$ estilo parental promove uma relação parento-filial estável e está associado a duas dimensões (MARTINS et al., 2014).

Nesse sentido, duas dimensões são fortemente consideradas em relação aos estilos parentais, sendo elas: o calor e o rigor parental. O calor parental está relacionado ao apoio, afeto, diálogo e a dimensão do rigor parental está associada ao controle, supervisão, autoridade e nomas de comportamento (NGUYEN et al., 2020).

Portanto, considerando as práticas parentais de uso dessas duas dimensões, quatro estilos parentais são descritos: autoritativo, autoritário, indulgente e negligente (MARTINEZ et al., 2020).

Diante disso, o estilo autoritativo está associado a práticas com alto calor e com

alto rigor, podendo contar com punições e castigo. $O$ autoritário diferencia-se no quesito calor, que não está presente, contando apenas com alto rigor (MARTINEZ et al., 2020).

Entretanto, o estilo indulgente está mais ligado a práticas de alta cordialidade, porém não conta com práticas de rigor comportamental. $O$ estilo negligente são práticas com a falta de calor e de rigor (MARTINEZ et al., 2020). Esses estilos são o modelo base, porém os estudos mostram variações entre sociedades e o tempo (NEVES, 2018).

Contudo, como citado por Neves (2018), as práticas parentais diversificam ao longo do tempo na sociedade, estando de acordo com novos paradigmas e estruturas. Essa diversificação aparenta estar ligada às crenças sociais do que é considerado uma boa parentalidade (NEVES, 2018).

Sendo assim, Martinez et al. (2020), foram capazes de apontar três estágios históricos acerca dos estilos parentais. O primeiro estágio, no início do século XX contava com o estilo parental autoritário como o ideal. Ao final do século, os estudiosos apontavam o estilo autoritativo como o ideal (segundo estágio).

$\mathrm{E}$ apenas, na era digital e nos mais atuais estudos (terceiro estágio), o estilo indulgente passou a mostrar-se com melhores práticas (MARTINEZ et al., 2020). Os

diferentes estágios dos estilos parentais estão fortemente associados ao desenvolvimento integral (habilidades cognitivas e não cognitivas) da criança (GARCIA; SERRA, 2019). 
Nesse sentido, o desenvolvimento de habilidades é dinâmico. Habilidades cognitivas e não cognitivas estão interligadas e não dependem apenas da genética, mas baseia-se nas vivências (HERNÁNDEZ-ALAVA; POPLI, 2017).

A saber, habilidades não cognitivas compreende como a categoria de destrezas como: habilidades sociais, regulação emocional, entre outros (LIPNEVICH; ROBERTS, 2012).

Desse modo, a junção das categorias de habilidades sociais e habilidades emocionais deram sentido ao construto das competências socioemocionais (MARIN et al., 2017). Portanto, é imprescindível conhecer a definição de Inteligência Emocional (IE) para melhor compreender as competências socioemocionais (MARIN et al., 2017).

Segundo Daniel Goleman (2012), IE é 'a capacidade de identificar nossos próprios sentimentos e dos outros, de nos motivarmos e gerirmos os impulsos dentro de nós e em nossos relacionamentos".

Eventualmente, as descobertas sobre IE têm destacado seu impacto em toda a vida do indivíduo. E mesmo sendo habilidades passiveis de treinamento, nota-se que as interações iniciais (pais-crianças) são cruciais (NGUYEN et al., 2020). Sendo o estilo parental mediador dessas interações (BAKER, 2017).

Assim como, Garcia e Serra (2019), trouxeram em seus estudos, as práticas parentais têm impactos em toda a vida do indivíduo, não se resumindo ao desenvolvimento infantojuvenil.

Diante disso, um consenso teórico acerca do estilo parental capaz de promover o melhor desenvolvimento das competências socioemocionais, pode gerar alterações de paradigmas familiares e educacionais. Podendo construir conscientização e intervenções precoces acerca do bem-estar integral dos pais e das crianças de maneira preventiva.

Por isso, o objetivo do presente estudo foi realizar uma revisão integrativa da literatura a fim de compreender a relação entre parentalidade e o desenvolvimento socioemocional.

\section{MATERIAL E MÉTODOS}

Foi feito um levantamento da literatura em abril de 2021, nas bases de dados Periódicos CAPES, Google Acadêmico e Pubmed. Os descritores utilizados foram os seguintes: 'Poder Familiar" AND 'Desenvolvimento Infantil" AND 'Inteligência emocional" e, em inglês, "Parenting AND 'Child Development" AND "Emotional Intelligence"em todas as bases de dados. Foram selecionados $\mathrm{x}$ artigos sendo incluídos segundo os critérios de elegibilidade conforme a Figura I. Os critérios de inclusão foram: artigos nos idiomas inglês, espanhol e português, nos últimos cinco anos, envolvendo o conhecimento sobre o desenvolvimento socioemocional e a contribuição dos estilos parentais para habilidades como autonomia, interações sociais e regulação emocional - Parentalidade e Desenvolvimento socioemocional. Os critérios de exclusão foram artigos de revisão de literatura ou metanálise. 

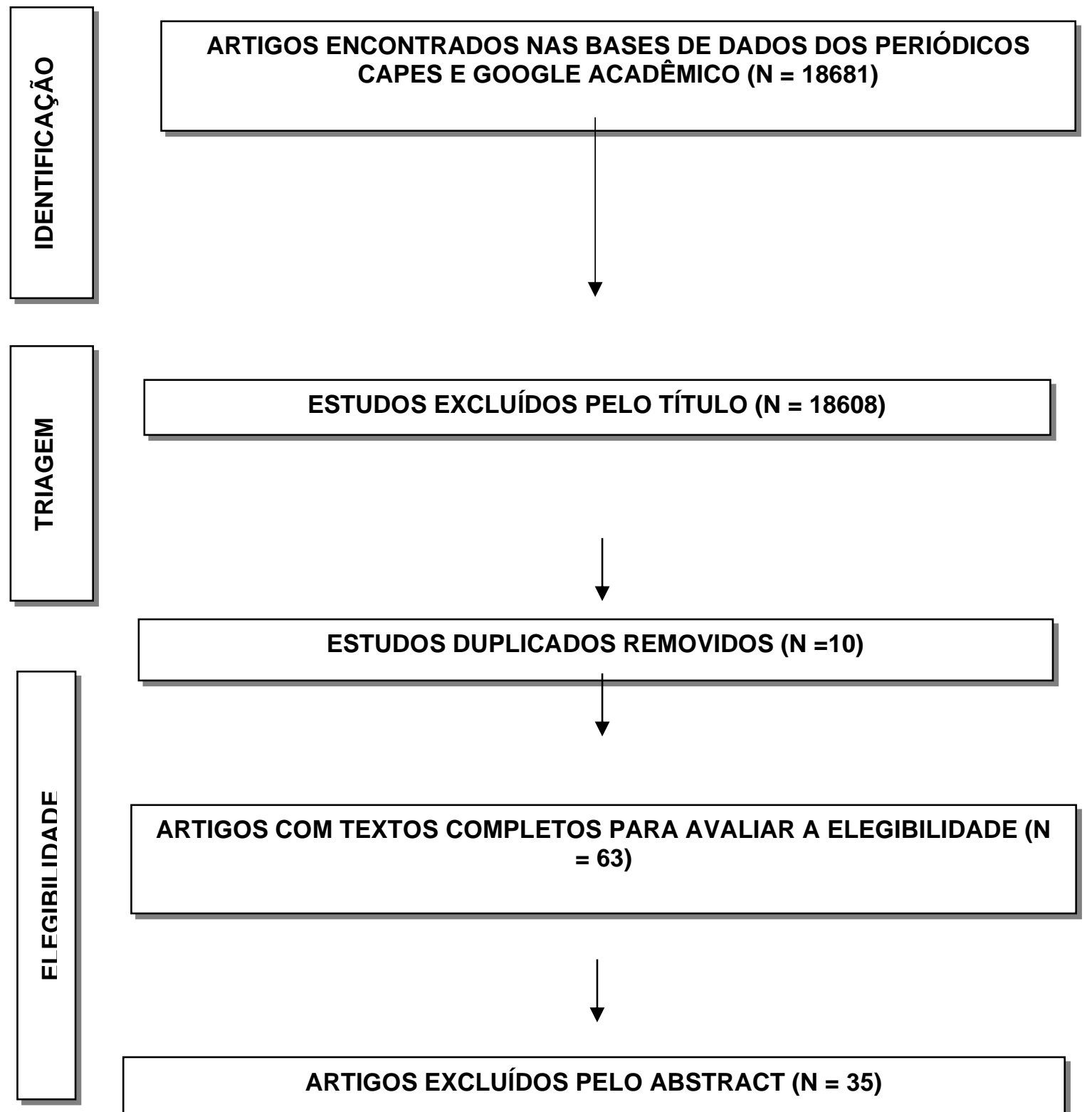
$=63$ )

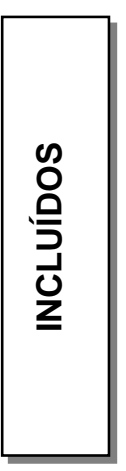

ARTIGOS EXCLUÍDOS A PARTIR DA LEITURA DO TEXTO COMPLETO ( $\mathrm{N}=$ 15)

ARTIGOS EXCLUÍDOS PELO ABSTRACT ( $\mathrm{N}=35)$

FIGURA I. FLUXOGRAMA E CRITÉRIOS DE SELEÇÃO E INCLUSÃo DOS ARTIGOS 
Tabela I - Demonstrativo dos artigos que integram a Revisão Integrativa

\begin{tabular}{|c|c|c|c|c|c|c|}
\hline$\# N$ & Data & Título & Autores & Periódico & Objetivos & Resultados \\
\hline I & 2020 & $\begin{array}{l}\text { Parenting } \\
\text { Styles, } \\
\text { Internalization } \\
\text { of Values and } \\
\text { Self-Esteem: A } \\
\text { Cross-Cultural } \\
\text { Study in Spain, } \\
\text { Portugal and } \\
\text { Brazil }\end{array}$ & $\begin{array}{l}\text { Isabel Martinez; } \\
\text { Fernando } \\
\text { Garcia; } \\
\text { Feliciano Veiga; } \\
\text { Oscar F. Garcia; } \\
\text { Yara Rodrigues; } \\
\text { Emilia Serra. }\end{array}$ & $\begin{array}{l}\text { International Journal } \\
\text { of Environmental } \\
\text { Research and Public } \\
\text { Health }\end{array}$ & $\begin{array}{l}\text { Analisar o impacto dos estilos } \\
\text { parentais na autoestima dos } \\
\text { adolescentes e internalização de } \\
\text { valores sociais em três países, } \\
\text { Espanha, Portugal e Brasil. }\end{array}$ & $\begin{array}{l}\text { Dos estilos parentais analisados, a parentalidade } \\
\text { indulgente e autoritativa mostraram maior impacto } \\
\text { na autoestima e internalização dos valores sociais nos } \\
\text { três países. Notou-se ainda que a influência da } \\
\text { parentalidade sobre a autoestima e os valores } \\
\text { acontece independente das diferenças culturais, o } \\
\text { sexo e a idade dos participantes. }\end{array}$ \\
\hline 2 & 2020 & $\begin{array}{l}\text { Perceived } \\
\text { Parenting } \\
\text { Styles and } \\
\text { Emotional } \\
\text { Intelligence } \\
\text { Among } \\
\text { Adolescents in } \\
\text { Vietnam }\end{array}$ & $\begin{array}{l}\text { Quynh-Anh N } \\
\text { Nguyen; Thach } \\
\text { D Tran; Tu- } \\
\text { Anh Tran; TA } \\
\text { Nguyen; Jane } \\
\text { Fisher. }\end{array}$ & $\begin{array}{l}\text { The Family Journal: } \\
\text { Counseling and } \\
\text { Therapy for Couples } \\
\text { and Families }\end{array}$ & $\begin{array}{l}\text { Examinar a associação entre estilos } \\
\text { parentais e Inteligência Emocional } \\
\text { entre adolescentes vietnamitas. }\end{array}$ & $\begin{array}{l}\text { Este estudo confirma o impacto dos estilos parentais } \\
\text { na Inteligência Emocional (IE). O calor dos pais, } \\
\text { presente nos estilos indulgente e autoritativo foi } \\
\text { associado a maior inteligência emocional, enquanto } \\
\text { maior autoritarismo e rigor foram associados a } \\
\text { menor inteligência emocional. O calor e o cuidado da } \\
\text { mãe e do pai beneficiarão o desenvolvimento } \\
\text { emocional de seus filhos no Vietnã. }\end{array}$ \\
\hline 3 & 2019 & $\begin{array}{l}\text { Raising } \\
\text { Children with } \\
\text { Poor School } \\
\text { Performance: } \\
\text { Parenting } \\
\text { Styles and } \\
\text { Short and Long- } \\
\text { Term } \\
\text { Consequences } \\
\text { for Adolescent } \\
\text { and Adult } \\
\text { Development }\end{array}$ & $\begin{array}{l}\text { Oscar F. Garcia; } \\
\text { Emilia Serra. }\end{array}$ & $\begin{array}{l}\text { International Journal } \\
\text { of Environmental } \\
\text { Research and Public } \\
\text { Health }\end{array}$ & $\begin{array}{l}\text { Examinar os correlatos dos estilos } \\
\text { parentais como: autoritativo, } \\
\text { indulgente, autoritário e negligente } \\
\text { na socialização de curto e longo prazo } \\
\text { em adolescentes e adultos. }\end{array}$ & 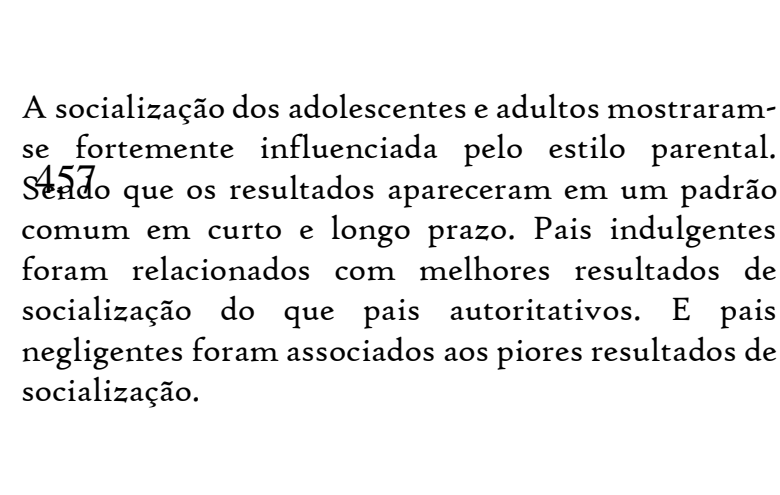 \\
\hline
\end{tabular}


The relative

of Maria

practices Gugliandolo; Stella

emotional

intelligence in an

Italian adolesce

sample

Parenting Style

and Reactive and

2018 Proactive

Adolescent

Violence: Evidence

from Spain

The relationship

between parenting

betyles parenting

Gugliandolo; Stella
Mavroveli;

Francesca

British Journal

uzzocrea

Developmental

Psychology

intelligence

kindergarten

children

Estilos Educativo

Parentais

Desenvolvimento

esonter

Majdi Adheisat;

Moreno-

iméne

gio

International Journal of

Environmental Research

de Competências

Iㅇ

em crianças do $\mathrm{I}^{-}$

Ciclo do Ensino

Básico
Early Child Investigar a relação entre os estilos em Governatorato de Zarqa II, Jordânia. parentais e inteligência emocional de uma

As variáveis das práticas parentais (tanto de apoio quanto de controle psicológico) foi relacionado ao traço de Inteligência Emocional. E os resultados mostraram que as práticas com alto controle psicológico foram preditoras negativas para a IE, principalmente, em meninos.

Anlisar a relação entre os estilos Parentais autoritativo, indulgente, autoritário e negligente - e a violência reativa e proativa entre pares na escola.

Os resultados sugeriram que o estilo parental autoritário está relacionado a maior envolvimento em comportamentos violentos com ações proativos e reativos. Observou-se que adolescentes com pais indulgentes apresentaram escores mais baixos na violência proativa.

Os resultados mostraram uma relação estatisticamente significativa e positiva entre os estilos parentais democráticos e inteligência emocional em todos os seus domínios, e mostrou uma relação estatisticamente significativa e negativa entre os estilos autoritativos e permissivos e inteligência emocional.

Os resultados mostraram que as competências socioemocionais das crianças 430 fortemente influenciadas pelo estilo educativo parental. Estilos permisivo mostrou correlaço negativa, princivalme dimensão adaptabilidade. 
Revista Ibero- Americana de Humanidades, Ciências e Educação- REASE open $\mathbf{\text { Access }}$

Children's

Development and Mónica Hernández-

Parental Input:

Evidence From the

UK Millen
Estimar uma dinâmica do modelo fatorial no desenvolvimento infantil.

\section{Alava; Gurleen Demography
Popli.}

Dissertação apresentada ao

Estilos Parentais e Vanessa Sá; Ligia programa de Pós Graduação Compreensão Maria Santos em Ciências Sociais Emocional de Monteiro; Patrícia Humanas da Instituto Emocional de Arriaga; Universitário de Lisboa Crianças em Idade Arriaga;
Pré-escolar

Father-Son

Relationships in

Ethnically Diverse

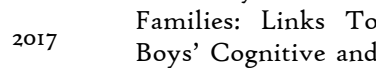

Social Emotional

Development in

Preschool
Claire E. Baker
Analisar as relações entre os estilos parentais e a compreensão emocional das crianças, controlando as variáveis sociodemográficas dos pais (idade e habilitações literárias) e da criança (idade, sexo).

Conceituar o envolvimento do pai como uma construção multidimensional - incluindo calor, controle / disciplina e estimulação da aprendizagem em casa - e examinados associações entre o envolvimento do pai durante a primeira infância e $\quad$ o
desenvolvimento cognitivo e social $e$
As práticas parentais em forma de atividades apresentaram significativo efeito no desenvolvimento infantil. $O$ investimento parental demonstrou efeito também na autoprodutividade, desenvolvimento cognitivo e não-cognitivo.

Este estudo mostrou que o calor parental e a estimulação dos pais em casa foram associados positivamente ao desenvolvimento de habilidades cognitivas e nãocognitivas. Sem diferenciações nos grupos raciais estudados.
Os resultados indicaram que as variáveis sociodemográficas dos progenitores não se encontram associadas aos Estilos Parentais, nem às competências emocionais das crianças. emocional de seus filhos na pré-escola.

Journal of Child and
Family Studies
Parental Behaviours

Predicting Early

Childhood

Early

a Meta-Analysis

\section{Debora S. Valcan \\ Debora \\ Deborah Davis; \\ Davis; Educational}

Psychology

Determinar a força da relação entre vários comportamentos parentais e Funções Executivas em crianças de 0 a 8 anos.
Os reftif 9 dos revelaram associações entre os comportamentos cognitivos dos pais e as Funções Executivas foram significativamente moderadas pela idade da criança, com as crianças mais novas mostrando um tamanho de efeito mais forte, nquanto os comportamentos parentais positivos e negativos mostraram uma associação estável com as Funções Executivas ao longo das idades. 
Revista Ibero- Americana de Humanidades, Ciências e Educação- REASE

\section{The Association}

between Parenting Zrinka Sosic-Vasic,

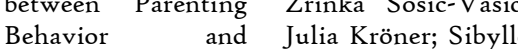

Executive Schneider; Nenad Frontiers in Psychology

Functioning in Vasic; Manfred

Children and Young Spitzer; Judith Streb.

Adolescents

The Development of

rosocial Behaviou

in Early Childhood:

Contributions of

Self- Regulation

Kate E. Williams;

International Journal Early Childhood
Investigar um possível fator que poderia se correlacionar com a Função Executiva de crianças em idade escolar crianças e adolescentes: comportamento parental.

Donna Berthelsen.

Considerar o papel das práticas parentais e da autorregulação precoce no comportamento prósocial das crianças no início da escola.
Os resultados mostram associações significativas entre vários comportamentos arentais e Funções Executivas dos filhos. Essas correlações entre o comportamento parental e Função Executiva permaneceram significativas, apesar da idade da criança, educação materna, renda familiar e desempenho básico.

A educação materna e paterna não hostil e o calor humano (estilo indulgente) fizeram contribuições indiretas significativas para o desenvolvimento pró-social posterior, influenciando a autorregulação precoce das crianças. 


\section{DISCUSSÃO}

O objetivo do presente artigo foi realizar uma revisão integrativa da literatura a fim de compreender os estilos parentais e os seus impactos no desenvolvimento socioemocional a partir das exigências e vivências da sociedade atual.

Sendo assim, como citado por Neves e Pires (2018), as práticas pautadas na afetividade e no diálogo tem ganhado espaço na sociedade. Apontando, os estilos autoritativos e indulgentes como agentes ativos no desenvolvimento infantojuvenil.

Neste sentido, adolescentes com pais indulgentes e autoritativos mostraram maior autoestima nas cinco áreas analisadas, acadêmica, emocional, familiar, física e social. Sendo que o rigor do estilo autoritativo, trouxe resultado inferior em relação a área social (MARTINEZ et al., 2020).

Para tanto, os estilos parentais autoritário e negligente demonstraram índices de baixa autoestima nos adolescentes nas cinco áreas observadas (MARTINEZ et al., 2020).

Ainda avaliando adolescentes, Nguyen et al. (2020), notaram que os Vietnamitas apresentaram resultados inferiores nas avaliações de Inteligência Emocional (IE), em destaque na subescala para confiança em si mesmo, em comparação com jovens gregos.

Isso pode se dar ao fato de os jovens vietnamitas estarem expostos a estilos parentais com mais rigor e pouco calor afetivo (autoritário), enquanto os jovens gregos foram expostos a práticas parentais com mais calor afetivo (indulgente e autoritativo) (NGUYEN et al., 2020).

A saber, os estilos parentais têm efeitos no desenvolvimento social, não apenas a curto prazo, mas a longo prazo (GARCIA; SERRA, 2019).

$\mathrm{Na}$ medida em que, Garcia e Serra (2019) apontam que mesmo de maneira decrescente em relação a idade, adultos e idosos criados com estilos parentais indulgentes e autoritativos apresentaram níveis mais elevados de IE através da autoestima, auto competência, competência social e empatia.

Enquanto, adultos e idosos que relataram pais autoritários ou negligentes mostraram baixa autoestima e níveis inferiores de auto competência, competência social e empatia (GARCIA; SERRA, 2019).

Não só isso, mas Garcia e Serra (2019), apontaram que os níveis de inteligência emocional dos adultos e idosos eram inferiores aos dos adolescentes em famílias com os mesmos estilos parentais (autoritário e negligente). Mostrando que os efeitos são prolongados e as poucas habilidades desenvolvidas podem decair ao longo do tempo.

Todavia, Gugliandolo et al., (2019) trazem que o período para os melhores resultados da Inteligência Emocional é na pré-adolescência, pois é normal na transição o adolescente perder a confiança em suas capacidades emocionais.

Para isso, os aspectos parentais que se destacaram como preditores positivos na Inteligência Emocional dos pré-adolescentes foram o apoio a autonomia materno e o envolvimento afetivo paterno (GUGLIANDOLO et al., 2019). 
Diante disso, o principal preditor negativo descrito foi o controle psicológico parental, onde os pais fazem seus filhos dependentes emocionais e psicológicos (GUGLIANDOLO et al., 2019).

Entre os vários âmbitos da inteligência emocional e da inteligência social trazidos em associação com a parentalidade, Moreno-Ruiz et al., (2018) alega três esferas da violência (pura, reativa e proativa).

Desse modo, a violência obteve maior grau nos estilos parentais autoritário e negligente. Mais uma vez, o estilo parental indulgente apresentou melhores escores em associação ao desenvolvimento de habilidades sociais (MORENO-RUIZ et al., 2018)

Ademais, com crianças no jardim da infância Al-Elaimat, (2018) demonstrou que o estilo parental indulgente (nomeado como democrático) favoreceu a inteligência emocional através de um ambiente seguro, diálogo e compromisso com as regras.

Porém, as ameaças, punições corporais e falta de explicações às crianças relacionou os estilos permissivos e autoritativos com problemas comportamentais infantis como indisciplina, baixa autoestima e pouca regulação emocional (AL-ELAIMAT; ADHEISAT; ALOMYAN, 2018).

Segundo Neves e Pires (2018), o aumento na adoção de estilos parentais autoritativas $e$ indulgentes tem relação com a mudança do paradigma e estrutura familiar ao longo do tempo na sociedade.

Com isso, sociedades que acreditavam que as práticas educacionais deveriam basear-se em punições e castigos tem repensado esse processo. Adotando práticas pautadas em afetividade, suporte emocional e diálogo com as necessidades dos filhos (NEVES, 2018).

Sabe-se que os primeiros anos da vida de uma criança repercute em todo o seu desenvolvimento. Não só estilo parental geral, mas algumas práticas específicas demonstraram maior valência no desenvolvimento de habilidades cognitivas e não cognitivas (HERNÁNDEZ-ALAVA; POPLI, 2017).

Desse modo, as vivências que fazem diferença no desenvolvimento das crianças são hábitos de rotina, ler para a criança, ajudá-la com as tarefas escolares nos anos de educação infantil. Tais vivências mostraram efeito imediato no desenvolvimento cognitivo e efeitos a longo prazo no desenvolvimento não cognitivo (HERNÁNDEZALAVA; POPLI, 2017).

Ainda sobre as práticas parentais positivas para o desenvolvimento socioemocional das crianças, temos o calor parental e a estimulação a aprendizagem como preditores para melhores resultados em testes de atenção e menores índices de envolvimento em comportamentos negativos (BAKER, 2017).

Além disso, o calor materno que promoveu processo atencional positivo e desenvolvimento de regulação emocional aos 2, 3 anos de idade, está também envolvido ao desenvolvimento do comportamento pró-social aos 6-7 anos de idade (WILLIAMS; BERTHELSEN, 2017).

Acerca do desenvolvimento emocional das crianças, Sá (2017), apontaram que estilos parentais autoritativos contribuem para a compreensão emocional (ler as expressões faciais dos outros), regulação emocional e tomada de decisões. 
Entretanto, o estilo parental permissivo pouco contribuiu para o desenvolvimento emocional das crianças, desde o processo de compreensão emocional. Tal dificuldade associada a falta de exigência traz uma ideia subentendida das emoções do cuidador (SÁ, 2017).

Enquanto isso, o estilo autoritário contribuiu para o desenvolvimento da compreensão emocional, mas apresentou baixos resultados na regulação emocional. Tendo em vista que tal estilo parental está associado a castigos e punições (SÁ, 2017).

De tal maneira, a parentalidade autoritária pode estar ligada a comportamentos de hostilidade e punição. Processos ligados a regulação emocional pobre, baixo envolvimento atencional, que levam a um não desenvolvimento de comportamentos pró-sociais (WILLIAMS; BERTHELSEN, 2017).

Para além do desenvolvimento socioemocional analisado através dos comportamentos infantis, há também um conjunto de funções neurológicas que estão associadas a competências socioemocionais, essas são as funções executivas (BRYDGES et al., 2012).

Nesse sentido, os estilos parentais demonstraram grandes impactos nas funções executivas das crianças. Famílias com práticas parentais positivas ofertam um ambiente seguro, motivador e encorajador, tornando a criança exploradora, confiante e resiliente (VALCAN; DAVIS; PINO-PASTERNAK, 2017).

Sendo assim, o ambiente seguro permite a exploração, pais calorosos encorajam seus filhos, suas emoções e otimismo. E o apoio e consideração aos problemas e soluções das crianças as tornam persistentes e menos impactadas aos fracassos (VALCAN; DAVIS; PINO-PASTERNAK, 2017).

Em contrapartida, estilos parentais com comportamentos negativos, como o negligente, estão associados a comportamentos hiperativos, ansiedade relacional e menor condições de regulação emocional (VALCAN; DAVIS; PINOPASTERNAK, 2017).

A saber, estilos parentais inconsistentes promoveram maior taxas de erros em testagens das funções executivas infantojuvenis. Tais resultados promoveram a análise de que essas crianças apresentaram atraso no desenvolvimento da autonomia, menor autorregulação emocional e baixa flexibilidade ao erro (SOSIC-VASIC et al., 2017).

Contudo, formas de parentalidade responsáveis, trouxeram resultados com maior adequação comportamental, liberdade nas escolhas das ações e consciência das consequências de tais ações (SOSIC-VASIC et al., 2017).

Inegavelmente, os resultados encontrados nessa revisão devem ser utilizados como base para futuras pesquisas, pois os dados obtidos mostram que existem práticas parentais que promovem o desenvolvimento e formação de indivíduos mais resilientes, com alta autoestima, melhor regulação emocional e empatia. Dessa forma são necessárias políticas que permitam aos pais a disponibilidade afetiva necessária aos cuidados integrais da criança. 


\section{CONCLUSÃO}

O objetivo do presente artigo foi realizar uma revisão integrativa da literatura a fim de compreender qual estilo parental proporciona práticas que estimulam o desenvolvimento das habilidades socioemocionais. Diante do exposto pode-se notar que dois estilos parentais são citados com práticas capazes de incitar a inteligência emocional e social nas crianças.

Portanto, temos resultados positivos para os estilos parentais indulgente e autoritativo. Sendo o estilo indulgente, pautado em calor afetivo, envolvimento emocional, diálogo, respeito as regras e acolhimento as necessidades e emoções da criança, obtendo índices ainda melhores.

Ainda assim, o estilo autoritativo que apresenta práticas próximas ao indulgente não obteve os melhores parâmetros por apresentar práticas de controle e punições.

Não só isso, os estilos parentais que apresentaram práticas favoráveis ao desenvolvimento das competências socioemocionais, estão associados também a escores elevados nas funções executivas.

Entretanto, tal fato assinala um desenvolvimento neurológico contundente, anunciando melhores condições cognitivas e não cognitivas ao longo da vida do indivíduo.

É provável que, o apontamento de dois estilos parentais trazidos como ideais para o atual momento, seja indicativo de um processo de mudança social. Pois em diferentes momentos históricos, diferentes estilos parentais eram anunciados como ideias.

Sendo assim, as práticas parentais que promovem um desenvolvimento saudável das habilidades cognitivas e não cognitivas em todo o ciclo de vida do ser humano, são pautadas no respeito, afetividade, acolhimento, ambiente seguro e liberdade de expressão.

\section{REFERÊNCIAS}

AL-ELAIMAT, A.; ADHEISAT, M.; ALOMYAN, H. The relationship between parenting styles and emotional intelligence of kindergarten children. Early Child Development and Care, v. 190, n. 4, p. 478-488, 2018.

BAKER, C. E. Father-Son Relationships in Ethnically Diverse Families: Links To Boys' Cognitive and Social Emotional Development in Preschool. Journal of Child and Family Studies, v. 26, n. 8, p. 2335-2345, 2017.

BRYDGES, C. R. et al. A unitary executive function predicts intelligence in children. Intelligence, v. 40, n. 5, p. 458-469, 2012.

GARCIA, O. F.; SERRA, E. Raising children with poor school performance: parenting styles and short-and long-term consequences for adolescent and adult development. International Journal of Environmental Research and Public Health, v. I6, n. 7, p. I4-17, 2019. 
GUGLIANDOLO, M. C. et al. The relative contribution of parenting practices in predicting trait emotional intelligence in an Italian adolescent sample. British Journal of Developmental Psychology, v. 37, n. 4, p. 585-599, 2019.

HERNÁNDEZ-ALAVA, M.; POPLI, G. Children's Development and Parental Input: Evidence From the UK Millennium Cohort Study. Demography, v. 54, n. 2, p. 485-5II, 2017.

LIPNEVICH, A. A.; ROBERTS, R. D. Noncognitive skills in education: Emerging research and applications in a variety of international contexts. Learning and Individual Differences, v. 22, n. 2, p. 173-177, 2012.

MARIN, A. H. et al. Social-emotional competence: concepts and associated instruments. Revista Brasileira de Terapias Cognitivas, v. 13, n. 2, p. 92-103, 2017.

MARTINEZ, I. et al. Parenting styles, internalization of values and self-esteem: A cross-cultural study in Spain, Portugal and Brazil. International Journal of Environmental Research and Public Health, v. 17, n. 7, p. I-I8, 2020.

MORENO-RUIZ, D. et al. Parenting style and reactive and proactive adolescent violence: evidence from Spain. International Journal of Environmental Research and Public Health, v. 15, n. 12, 2018.

NEVES, C. S. L. Estilos Educativos Parentais e Desenvolvimento de Competências Sócio-Emocionais em crianças do $\mathrm{I}^{\circ}$ Ciclo do Ensino Básico. [s.l: s.n.].

NGUYEN, Q. A. N. et al. Perceived Parenting Styles and Emotional Intelligence Among Adolescents in Vietnam. Family Journal, v. 28, n. 4, p. 441-454, 2020.

PIRES COLTRO, B.; PARAVENTI, L.; VIEIRA, M. L. Relações entre Parentalidade e Apoio Social: Revisão Integrativa de Literatura. Contextos Clínicos, v. 13, n. I, 23 jul. 2020.

RICARDO PRADO MARTINS et al. PRÁTICAS PARENTAIS: ASSOCIAÇÕES COM DESEMPENHO ESCOLAR E HABILIDADES SOCIAIS. Psicologia Argumento, v. 32, n. 78, p. 89-100, 24 nov. 2017.

SÁ, V. Estilos Parentais e Compreensão Emocional de Crianças Em Idade Pré Escolar. [s.l: s.n.].

SOSIC-VASIC, Z et al. The association between parenting behavior and executive functioning in children and young adolescents. Frontiers in Psychology, v. 8, n. MAR, p. I-8, 2017.

VALCAN, D. S.; DAVIS, H.; PINO-PASTERNAK, D. Parental Behaviours Predicting Early Childhood Executive Functions: a Meta-Analysis. Educational Psychology Review, v. 30, n. 3, p. 6o7-649, 2017.

WILLIAMS, K. E.; BERTHELSEN, D. The Development of Prosocial Behaviour in Early Childhood: Contributions of Early Parenting and Self-Regulation. International Journal of Early Childhood, v. 49, n. I, p. 73-94, 2017. 